\title{
WISC-III e WAIS-III: Alterações nas Versões Originais Americanas Decorrentes das Adaptações para Uso no Brasil
}

\author{
Elizabeth do Nascimento ${ }^{12}$ \\ Universidade Federal de Minas Gerais \\ Vera Lúcia Marques de Figueiredo ${ }^{3}$ \\ Universidade Católica de Pelotas, Rio Grande do Sul
}

\begin{abstract}
Resumo
O presente trabalho apresenta alterações efetuadas entre as versões americanas dos testes WISC-III e WAIS-III e as adaptadas para uso no Brasil. As análises empíricas realizadas com base nas amostras de adaptação, validação e normatização do WISC-III (801 crianças residentes na zona urbana da cidade de Pelotas/RS) e do WAIS-III (788 participantes adolescentes e adultos residentes na região metropolitana de Belo Horizonte/ $\mathrm{MG}$ ) acarretaram em alterações nas versões originais dos testes, no que se refere: aos conteúdos de alguns ítens dos subtestes verbais; à ordem de apresentação dos itens; aos tempos limites e à concessão de bônus; aos critérios de início e suspensão da aplicação e; aos grupos etários para os quais foram estabelecidas normas. Os resultados reforçam a importância de se proceder amplos estudos com instrumentos criados em contextos diferentes daqueles em que serão utilizados.

Palavraschave: WISC-III; WAIS-III; adaptação de testes; inteligência.
\end{abstract}

\section{W ISC-III and WAIS-III: Alterations in the Current American \\ Original Versions of the Adaptations for Use in Brazil}

\begin{abstract}
This paper presents the main alterations that were made in the Brazilian versions of the tests WISC-III and WAIS-III. Empirical analyses were based on a sample of 801 children living in the urban area of Pelotas-RS and 788 adolescents and adults living the metropolitan area of Belo Horizonte MG and they resulted in changes in the original versions of the tests, with respect to: the content of some items of the verbal scale; the order of presentation of the items of the scales; the time limit and bonus concession; the criteria to start and discontinue the application; the establishment of norms for age. In spite of the fact that each study was restricted to one urban area of the country, their results reinforce the importance of adaptation and validation studies of instruments that have been developed in a context that is different from those in which they will be used.

Keywords: WISC-III; WAIS-III; test adaptation; intelligence.
\end{abstract}

Elaboradas para a avaliação de habilidades cognitivas, as Escalas Wechsler de Inteligência foram desenvolvidas ao longo de várias décadas. A primeira forma foi publicada em 1939 - a Escala de Inteligência WechslerBellevue - elaborada com a finalidade de oferecer um teste apropriado para a avaliação da inteligência de adultos. Os testes até então utilizados tinham sido, originalmente, construídos para a avaliação de crianças, o que gerava problemas tanto em relação à administração, quanto ao conteúdo e à correção. A composição da Escala (incluindo subtestes verbais e de execução) e a apresentação dos

\footnotetext{
${ }^{1}$ Endereço para correspondência: Universidade Federal de Minas Gerais, FAFICH - Departamento de Psicologia - Sala 4048, Av. Antônio Carlos, 6627, 31270-901, Belo Horizonte, MG. Fone: (31) 3499-6275. E-mail: enasci@gold.com.br

${ }^{2} \mathrm{O}$ projeto contou com o apoio institucional da UFMG e da CAPES.

${ }^{3} \mathrm{O}$ projeto contou com o apoio institucional da UCPel e do CNPq.
}

resultados em QI de desvio (baseados nas mesmas características de distribuição para todas as idades) representaram inovações importantes no contexto da avaliação da inteligência. Em 1949, surgiu a extensão para crianças em idade escolar (WISC), e em 1967, a escala para crianças em idade préescolar (WPPSI).

As três escalas mantêm a mesma estrutura, porém apresentam variantes adequadas para cada faixa etária. Revisões foram e continuam sendo realizadas ao longo de cada década, resultando versões mais atualizadas, conforme pode ser observado na Tabela 1.

As revisões das Escalas Wechsler, realizadas ao longo de décadas, tiveram como propósito aprimorá-las tanto do ponto de vista teórico quanto prático. Além da atualização denormas, as revisões proporcionaram a inclusão de novos subtestes, substituição deitens obsoletos, alteraçõesnas regras depontuação eobtenção denovos resultados. Cabedestacar que, em 1999, foi publicada uma forma reduzida dasEscalas Wechsler (WASI) que tem como finalidade proporcionar 
Tabela 1

Escalas Wechsler deInteligência segundo o Ano dePublicação eFaixa Etária a queseD estinam.

Escala

Wechsler-Bellevue Intelligence Scale - Form I

Wechsler-Bellevue Intelligence Scale - Form II

Wechsler Intelligence Scale for Children

Wechsler Adult Intelligence Scale

Wechsler Preschool and Primary Scale of Intelligence

Wechsler Intelligence Scale for Children- Revised

Wechsler Adult Intelligence Scale - Revised

Wechsler Preschool and Primary Scale of Intelligence - Revised

Wechsler Intelligence Scale for Children- Third edition

Wechsler Adult Intelligence Scale - Third edition

Wechsler Abbreviated Scale of Intelligence

$\begin{array}{cr}\text { Ano de publicação } & \text { Faixa etária } \\ 1939 & 7-69 \\ 1946 & 10-79 \\ 1949 & 5-15 \\ 1955 & 16-64 \\ 1967 & 4-6,5 \\ 1974 & 6-16 \\ 1981 & 16-74 \\ 1989 & 3-7,5 \\ 1991 & 6-16 \\ 1997 & 16-89 \\ 1999 & 6-89\end{array}$

uma avaliação breve da inteligência deindivíduos de todas as faixas etárias.

A concepção de inteligência de David Wechsler que norteou a construção de escalas que levam o seu nome manteve-se a mesma ao longo de toda a sua trajetória científica. Para ele, a inteligência, enquanto construto hipotético, refere-se à "capacidade conjunta ou global do indivíduo para agir com finalidade, pensar racionalmente e lidar efetivamente com seu meio ambiente" (Wechsler, 1944, p. 3). Wechsler a considerava global por caracterizar 0 comportamento do indivíduo como um todo, e conjunta por ser composta de capacidades qualitativamente diferenciáveis, mas não inteiramente independentes, sendo então, multifacetada e multideterminada. Em seus artigos, o autor faz questão deressaltar queinteligência ecapacidades intelectuaissão diferentes, poisainteligênciaéinferida segundo os modos como as capacidades se manifestam sob diferentes condições ecircunstâncias.

A utilização das Escalas Wechsler de Inteligência está voltada para os contextos clínico, psicoeducacional e de pesquisa, possibilitando a avaliação minuciosa das capacidades cognitivas de crianças, adolescentes e adultos. A aplicação das EscalasWechsler deInteligência éindividual e exigequeo profissional seja altamente treinado tanto para a aplicação quanto para a correção.

\section{W ISC-III e o WAIS-III}

Algumas alterações decorrentes das revisões dos testes WISC-R e WAIS-R foram comuns ao WISC-III e WAIS III. Dentre elas destacam-sea inclusão denovos subtestes e a possibilidade de sintetizar os resultados (além dos tradicionais QI Total, QI Verbal e QI de Execução) em quatro índices derivados das análises fatoriais. São eles: Compreensão Verbal, Organização Perceptual, Velocidade de Processamento e um quarto índice denominado
Resistênciaà Distração no WISC-III eMemória deTrabalho no WAIS-III. Esses quatro índices representam dimensões relevantes no conhecimento das capacidades cognitivas dos sujeitos examinados.

A terceira revisão da escala WISC foi editada em 1991, nos Estados Unidos (Tabela 1). Nesse teste foram incluídas algumas mudanças no material e nos procedimentos de aplicação na tentativa de torná-lo mais interessante para as crianças. Embora apresentevárias melhorias eum acréscimo substancial deitens, elemantém as características básicas do WISC e do WISC-R. Mais de 73\% dos itens do WISC-R foram mantidos na forma original ou tiveram mudanças insignificantes (Wechsler, 1991).

Dos 13 subtestes que compõem o WISC-III, 12 são os mesmos das edições anteriores e foi adicionado o novo subtesteProcurar Símbolos (medida da atenção evelocidade de processamento). Os subtestes medem diferentes capacidades e estão agrupados, como nas demais escalas de Wechsler, em um conjunto verbal de seis subtestes (Informação, Semelhanças, Aritmética, Vocabulário, Compreensão e Dígitos) e outro não-verbal ou de execução formado por sete subtestes (Completar Figuras, Arranjo de Figuras, Código, Cubos, Armar Objetos, Procurar Símbolos e Labirintos).

A principal razão para uma nova edição do WISC-R de 1974 foi a desatualização de suas normas americanas em aproximadamente 15 anos. Considerando os estudos de Flynn (1987), mostrando que a média do QI vem aumentando, no mínimo, em torno de3 pontos por década, principalmente, no que se refereà capacidade não-verbal, a utilização denormas desatualizadas produziriam resultados inflados.

O teste WAIS-III constitui a terceira edição do WAIS e foi publicado nos Estados Unidos da América, em 1997 (Tabela 1). Trata-se de uma versão aperfeiçoada do WAIS 
$\mathrm{R}$, não somente em relação ao conteúdo e formato dos itens, instruções, diminuição da influência do fator tempo limite e correção, mas também pela possibilidade que apresenta de investigar de forma detalhada e sistemática diferentes facetas do funcionamento cognitivo de adolescentes eadultos, ampliando assim sua utilidade clínica.

O WAIS-III mantém a mesma estrutura do WAIS-R e das outras escalas Wechsler de inteligência. Mais de 68\% dositens do WAISR (excluindo ositens do subtesteDígitos) foram mantidos, seja na forma original ou ligeiramente modificada. Dentre as alterações efetuadas, verifica-se que houve 0 aumento no número de itens em nove subtestes e um ligeiro decréscimo em apenas dois, quando comparado com o WAIS-R (The Psychological Corporation, 1997).

Três novos subtestes foram integrados ao WAISIII, a saber, Raciocínio Matricial (medida do processamento da informação visual e raciocínio abstrato), Seqüência de Números e Letras (medida da atenção e memória de trabalho) e Procurar Símbolos. Este último consiste em uma adaptação do já incluído no WISC-III. Desta forma, atualmente, a escala é composta de 14 subtestes, estando agrupados em dois conjuntos, com sete subtestes cada: verbal (Vocabulário, Semelhanças, Aritmética, Dígitos, Informação, Compreensão e Seqüência de Números e Letras) e de execução (Completar Figuras, Códigos, Cubos, Raciocínio Matricial, Arranjo deFiguras, Procurar Símbolos e Armar Objetos).

Embora o número de itens tenha aumentado nos testes WISC-III e WAIS-III em razão da inclusão de novos itens, o tempo de aplicação permanece em torno de 90 minutos para ambos. Em geral, os procedimentos de aplicação, pontuação, registro e interpretação dos resultados são os mesmos.

Do ponto de vista psicométrico, tanto o WISC-III quanto o WAIS-III apresentam características semelhantes às outras EscalasWechsler deInteligênciano quediz respeito à fidedignidade e validade, revelando-se, como os seus predecessores, em instrumentos adequados para a investigação dainteligência.

Instrumentos como as Escalas Wechsler de Inteligência, queestão entre os maisinvestigados eusados, são geralmente adaptados para outros países. A prática corrente de adaptar instrumentos desenvolvidos e normatizados em uma determinada cultura parauso em outra não está circunscrita somente a pesquisas transculturais, mas também, para uso intracultural (Hambleton \& Bollwark, 1991). Como afirmam Van de Vijver e Hambleton (1996) o processo de adaptação detestes psicológicosapresenta dificuldades, muitas das quais relacionadas às diferenças entre a cultura de origem do teste e a cultura para onde ele está sendo adaptado, sendo que, quanto maior a distância cultural, maior o número de dificuldades em se obter uma versão que seja equivalente.

As diretrizes estabelecidas pela Comissão Internacional de Testes (Hambleton, 1993, 1994; Muñiz, \& Hambleton, 1996; Van de Vijver \& Hambleton, 1996) constituem em importantes pontos de referência para a realização do processo deadaptação deum instrumento paraum contexto diferentedaqueleem quefoi construído. As recomendações preconizadas na área da construção e adaptação do teste indicam os procedimentos básicos relacionados ao processo de adaptação visando que o teste meça o mesmo construto, embora ajustado às peculiaridades da nova população. Essas recomendações foram norteadoras de algumas etapas das pesquisas de adaptação dos testes WISC-III e WAIS-III para uso no Brasil epermitem identificar que o processo de adaptação envolve muito mais que a tradução do instrumento e deve estar respaldado em investigações empíricas que o legitime.

\section{Método}

\section{Participantes}

Os dados aqui analisados pertencem aos estudos de adaptação, validação e normatização do WISC-III (Figueiredo, 2001) e do WAIS-III (Nascimento, 2000) para amostras brasileiras.

A amostra do WISC-III $(\mathrm{N}=801)$ foi constituída por crianças residentes na região sul do Brasil, representando em torno de 1\% da população geral de escolares da cidade de Pelotas. As idades variaram de 6 a 16 anos e foram agrupadas em seis faixas etárias $(6,7,8-9,10-11,12-13$ e 14-16 anos) com proporção similar de meninos (49\%) e meninas (51\%). As crianças selecionadas estavam matriculadas em 34 instituições de ensino público (84\%) e privado (16\%), localizadas tanto no centro como na periferia, representando $32 \%$ do total de escolas da zona urbana da cidade.

A amostra do WAISIII $(\mathrm{N}=788)$ foi constituída de indivíduos com idades entre 16 e 89 anos, delineada de forma a ser o mais representativa possível da população de Belo Horizonte/MG. Para tal, foram consideradas as seguintes variáveis para sua composição: idade, sexo e anos deestudo. A amostra ficou composta de oito grupos etários: $16-17,18-19,20-29,30-39,40-49$, 50-59, 60-64 e 65-89 anos. $\mathrm{O}$ número de participantes em cada grupo etário variou entre 83 e 118 sujeitos. Da mesma forma que na versão original americana do teste, considerou-se a variável anos de estudo. Nessa amostra, ela foi organizada em cinco categorias: 3 anos ou menos; 4 a 7 anos, 8 a 10 anos, 11 a 14 anos e mais de 15 anos de estudo. 


\section{Procedimentos}

As respectivas pesquisas de adaptação foram realizadas contemplando as seguintes etapas: tradução para a língua brasileira dos conteúdosverbais edasinstruções deaplicação ecorreção; análiseteórica dositens (juízesesemântica); coleta dedados; análiseempírica dos dados (análisedos parâmetros psicométricos dos itens e do teste como um todo) e estabelecimento das normas.

$\mathrm{Na}$ fase da coleta de dados, os participantes de cada uma das pesquisas foram testados individualmente após consentimento livre informado, sendo que, no caso das crianças, a participação foi condicionada à autorização dos responsáveis. Todos os examinadores foram treinados sistematicamente. No WISC-III, o tempo total de testagem de cada criança participante foi de 90 minutos, em média, enquanto no WAIS-III foi de 120 minutos. Os procedimentos de aplicação seguiram as orientações recomendadas nos manuais americanos dos respectivos testes, embora algumas modificações tenham sido feitas para consecução dos objetivos das pesquisas de adaptação e validação dos instrumentos. Os escores, obtidos pelos sujeitos, foram digitados num banco de dados que resultou nas matrizes que deram base às análises estatísticas.

Cabemencionar queno WISC-III, o subteste Labirintos não foi objeto de investigação; considerando seus baixos índices de intercorrelação com os demais subtestes, a baixa carga fatorial com o fator $g$ e, ainda, pelo subteste não ser relevante nem para a obtenção dos escores de Q Is nem para os dosíndicesfatoriais. Com a finalidadedeaperfeiçoar a apresentação dos estímulos do subtesteProcurar Símbolos, a pesquisadora acrescentou uma linha divisória entre os grupos de estímulos (padrão eprocura) com vistas a facilitar a discriminação dos símbolos dos dois conjuntos.

\section{Resultados}

Os resultados serão apresentados para cada um dos testes, contemplando as alterações nos seguintes tópicos: conteúdos e ordem de apresentação dositens nos subtestes; tempos limites finais, concessão debônus, critérios deinício e suspensão e grupos etários para o estabelecimento de normas.

\section{Conteúdos dos itens nos subtestes}

No processo de adaptação dos testes, as etapas de tradução (dositens e das instruções) e de análise teórica dos itens tiveram, entre outros objetivos, alcançar uma equivalência entre os itens nos dois contextos (americano e brasileiro).

Cabe destacar que embora os itens dos subtestes Completar Figuras e Arranjo de Figuras em ambos testes apresentem estímulos carregados defatores culturais, estudos preliminares realizados pelas autoras mostraram que tais itens revelaram ser adequados para uso no Brasil, segundo a avaliação dos sujeitos participantes das amostras pilotos.

No teste WISC-III, o subteste em que ocorreu o maior número de substituições foi Informação por se mostrar mais carregado de influências culturais. No subteste Aritmética, foram mantidos todos os valores numéricos propostos para os cálculos, modificando-se somente 0 sistema monetário (real em vez de dólar) e os nomes próprios utilizados nos problemas. Os nomes não usuais no idioma brasileiro foram substituídos por outros mais comuns. No problema 23, o objeto bicicleta foi substituído por rádio para propiciar maior coerência entre objeto e valor referido (Tabela 2).

No WAIS-III, os subtestes Vocabulário e Informação foram aqueles que apresentaram maior complexidade no processo de adaptação. No subteste Vocabulário, embora não tenha havido nenhuma alteração nositens, a principal dificuldade foi encontrar a melhor tradução para palavras, particularmente, 'fortitude', 'ominous' e 'encumber'. Como no WISC-III, no subteste Aritmética, foram realizadas modificações no sistema monetário enasunidades demedida (milhas para quilômetros) além de nomes fantasias, mantendo-se as operações matemáticas dos itens originais. 0 item 12 foi reformulado com o objetivo de encontrar umasituação concretano contexto brasileiro queenvolvesse o mesmo tipo de raciocínio do item original.

No WAIS-III, foi necessário alterar um termo no item original americano do subteste Semelhanças porque o par de cores (amarelo - verde) acarretou em uma restrição nas possibilidades derespostas, visto quemuitos dosparticipantes de estudos piloto se referiam diretamente às cores da bandeira nacional brasileira. No subteste Compreensão alterou-se o ditado da versão americana (item 18) por outro mais correnteno contexto brasileiro.

A Tabela 2 apresenta uma síntese dositens alterados nos testes WISC-III e WAIS-III.

Como pode ser observado na Tabela 2, o subteste Informação foi o que sofreu maior número de alterações em ambostestes. As mudanças efetuadas não se restringiram à substituição por conteúdos próprios ao contexto nacional. Modificações também ocorreram nositens com conteúdos gerais com o objetivo de torná-los mais fáceis, o que possibilitou estabelecer melhor equilíbrio no grau de dificuldade no subteste.

\section{Ordem de apresentação dos itens dos subtestes que compõem a escala}

Considerando que os itens dos testes que avaliam a capacidade intelectual devem ser apresentados em uma 
Tabela 2

Alterações nos Itens do WISC-III e WAIS-III

\begin{tabular}{|c|c|c|c|}
\hline Teste & Subteste & Item original* & Item adaptado \\
\hline \multirow[t]{8}{*}{$\overline{\text { W ISC-III }}$} & & 13. Who was Christopher Columbus? & 12. ..Pedro Álvares Cabral? \\
\hline & & 18. On what continent is Brazil? & 20. ... Canadá? \\
\hline & & 20. Who invented the electric light? & 18. ... o Avião? \\
\hline & Informação & 22. Who was Anne Frank? & 13. ... Monteiro Lobato? \\
\hline & & 25. What is the capital of Greece? & 16. ... França? \\
\hline & & 29. How far is it from London to New York? & 29. ... São Paulo e Lisboa? \\
\hline & & 30. What does turbentine come from? & 26. ... o Látex? \\
\hline & Aritmética & $\begin{array}{l}\text { 23. Becky bought a second-hand bicycle for } 28 \\
\text { dollar. She paid } 2 / 3 \text { of what the bicycle cost } \\
\text { new. How much did it cost new? }\end{array}$ & $\begin{array}{l}\text { 21. Beto comprou um rádio de } \\
\text { Segunda mão por } 28 \text { Reais. Ele pagou } \\
2 / 3 \text { do preço de um rádio novo. Qual } \\
\text { o preço de um rádio novo? }\end{array}$ \\
\hline
\end{tabular}

\begin{tabular}{ll}
\hline WAIS-III Semelhanças & 3. Yellow - Green \\
& $\begin{array}{l}\text { 12. Jesse bought } 6 \text { pieces of chocolate for } \$ \\
\text { 1.60. Na additional } 20 \text { cents sales tax was added } \\
\text { to this price. How much did he pay for each } \\
\text { chocolate including sales tax? }\end{array}$
\end{tabular}

3. Amarelo - Vermelho

12. José comprou 6 canetas para distribuir entre os colegas e pagou 16 reais. Para que fossem embrulhadas para presente, pagou mais 2 reais. Quanto ele pagou por cada caneta, incluindo o papel de presente?

8. Who wrote Hamlet?

11. ... Romeu e Julieta?

9. On what continent is Brazil?

8. ... Canadá?

10. Who was Martin Luther King, Jr.?

7. ... Tiradentes?

Informação 11. Who was President of the United States during the Civil War?

17. ... Brasil durante o período chamado Estado Novo?

18. Who painted the Sistine Chapel?

16. ... Monalisa?

28. Who wrote Faust?

15. ... Os Lusíadas?

Compreensão

18. What does this sayingmean: "Shallow brooks arenoisy."

7. ... “Cão que ladra não morde."

Nota. Fonte: * WISC-III = Wechsler (1991); WAIS-III = Wechsler (1997).

ordem crescente de dificuldade, procedeu-sealgumasanálises com vistas a identificar a ordenação dos itens para a população brasileira representada em cada uma das amostras utilizadas para adaptação dos testes WISC-III e WAIS-III. Cabe destacar que os subtestes Procurar Símbolos e Códigos não foram objeto de análise por serem testes de velocidade.

No WISC-III, para organizar o arranjo dos itens segundo a ordem crescente de dificuldade, foi utilizado o critério de percentual deacertos encontrado na amostra brasileira. Nenhuma mudança foi procedida em Dígitos. Nos demais subtestes, foi necessária a alteração na ordem de vários itens, com exceção de Cubos e Armar Objetos nos quais somente um item foi deslocado.

No WAIS-III, utilizou-se o parâmetro de dificuldade dos itens estabelecido pela Teoria de Resposta ao Item para estabelecer a ordem de apresentação dos itens em cada um dos subtestes na versão adaptada. Dentre os subtestes do WAIS-III, os itens dos subtestes Dígitos, Seqüência de Números e Letras e Armar Objetos mantiveram a mesma ordem da versão original. Cabe destacar que nos dois primeiros subtestes citados, embora a ordem deapresentação dositenstenha permanecido como na versão original, houve alteração na apresentação das tentativas. 
Pode-se concluir que a seqüência dos itens de cada subteste é determinada pelas experiências culturais do indivíduo, considerando que a ordenação dos itens nas amostras brasileiras configurou-se bastante diversa da determinada para os testes originais, principalmente nos subtestes verbais.

\section{Tempos limites finais para os subtestes de execução}

No WISC-III, observou-se que $80 \%$ das crianças que acertaram os itens completaram-nos dentro do tempo estipulado pelo manual original. Dessa forma, concluiuse que os tempos da versão americana do teste são adequados para a amostra pesquisada. Por essa razão, foram mantidos os mesmos tempos limites do teste original para todos os itens da versão brasileira, exceto para Armar Objetos, em que os tempos expandidos utilizados para a aplicação mantiveram-se na versão final, uma vez que não foram registradas as pontuações parciais obtidas dentro do tempo original, o que impossibilitou a revisão dos escores no caso de mudança do critério. Dessa forma, os tempos limitespara realização dositens do Armar Objetosficaram: 130 segundos para o item 1, 160 segundos para os itens 2 e 3 e 190 segundos para os itens 4 e 5.

No processo de adaptação do teste WAIS-III também investigou-se a adequação dos tempos limites originais dos subtestes que possuem esse parâmetro para aplicação. Os tempos limites estabelecidos na versão piloto do WAIS-III foram revistos com basenaamostra total. Os tempos limites finais para cada um dositens foram estabelecidos conforme o seguintecritério: tempo gasto por 70\% ou mais de pessoas que acertaram os itens investigados. Contudo, como no subtesteCompletar Figuras o tempo limiteé o mesmo para todos os itens, considerou-se a média geral de tempo gasto para responder corretamente todos os itens que 0 compõem. Os tempos limites dos subtestes Completar Figuras, Aritmética e Arranjo de Figuras não sofreram modificação. No subteste Cubos houve alteração dos temposlimites dositens 12, 13 e14, acarretando na expansão dos tempos originais em 10 segundos para os itens 12 e 13 e 30 segundos para o último item, passando então de 120 para 130 segundos e de 120 para 150 segundos, respectivamente. No subtesteArmar Objetos, o tempo limite da versão americana para o item 1 mostrou-se adequado para a adaptada, tendo sido então adotado. Com relação aos itens 2 e 3, embora os tempos limites originais tenham semostrado adequadospara a amostra brasileirainvestigada, não foram adotados pelos motivos já expostos em relação ao subteste no WISC-III. Quanto aositens 4 e 5, os tempos limites originais não foram suficientes para a amostra brasileira investigada. Dessa forma, nesse subteste ostempos limites ficaram expandidos, sendo que, nos itens 3, 4 e 5 a expansão foi de 60 segundos.

\section{Concessão de bônus para os itens cronometrados}

Todas as Escalas Wechsler de Inteligência incluem pontuações que refletem a qualidade e a velocidade de execução, sendo dado um ou mais pontos de bonificação pela realização correta e rápida do item (Wechsler, 1991). No W ISC-III, cinco subtestes recebem pontos adicionais: Código forma A, Arranjo de Figuras, Aritmética, Cubos e Armar Objetos. No WAIS-III, os subtestes em que os sujeitos recebem bonificações são os mesmos do WISCIII, com exceção do Código.

Na pesquisa de adaptação do WISC-III, as bonificações foram determinadas com base na distribuição das freqüências dos tempos de execução das crianças que realizaram a tarefa corretamente. Considerando que o teste original estabelece, para os subtestes Arranjo de Figuras, Cubos e Armar Objetos quatro categorias de bonificação, seguiu-se o mesmo critério assim como as próprias pontuações. Determinou-se como pontos de corte as freqüências alcançadas por 25\%,50\%, 75\% e 100\% da amostra. Assim, no subtesteArranjo deFiguras, por exemplo, o intervalo de tempo despendido por $25 \%$ da amostra ganhou 3 pontos de bonificação, passando-se seu escore de 2 pontos para 5 (2+3); o intervalo de tempo gasto por 26 a $50 \%$ da amostra obteve 2 pontos de bônus e o escore de 2 pontos passou para $4(2+2)$; o intervalo de tempo gasto por 51 a $75 \%$ da amostra ganhou 1 ponto debônus e o escore de 2 pontos passou para $3(2+1)$ e, finalmente, o intervalo de tempo gasto por 76 a 100\% da amostra determinou o bônus zero e os escores de 2 pontos obtidos neste intervalo de tempo permaneceram. Para o subteste Código forma A, não foram estabelecidos bônus para a amostra brasileira, uma vez que somente seis crianças entre 6 e 7 anos obtiveram 59 acertos ou mais nesse subteste, pontuações que receberiam pontos adicionais, segundo o manual original. Sem representatividade para analisar a distribuição de freqüência dos tempos, decidiu-se manter os critérios estabelecidos no manual original do teste para a pontuação do subteste Código forma A.

No WAIS-III, para concessão de bônus por tempo gasto nos subtestes Cubos e Aritmética foram adotados os mesmos critérios da versão original americana. Considerouse adequada a distribuição das percentagens de acertos nos intervalos detempos originais. Em relação ao subtesteArmar Objetos, dada a impossibilidade de reavaliar os tempos expandidos, a opção foi de alterar unicamente 0 primeiro intervalo detempo, expandindo ostemposlimites dositens 2, 3, 4 e 5 para osadotadosnaversão piloto. Como exemplo, no item 2 para concessão depontos pela execução completa 
e correta do item dentro do tempo estabelecido, passou de 36 a 120 segundosna versão original para 36 a 160 segundos na versão adaptada, embora a pontuação original tenha permanecido a mesma.

\section{Critérios de início e suspensão da aplicação dos subtestes}

a) Critériosdeiníåo

Tanto no WISC-III quanto no WAIS-III houve necessidade de alteração dos critérios de início da aplicação dos subtestes. O estabelecimento desses critérios foi conduzido considerando a percentagem de $95 \%$ ou mais de acerto nos itens (mesmo critério adotado nas versões originais dos testes), sendo que no WISC-III essa análise foi conduzida segundo cada faixa etária.

A Tabela 3 apresenta os critérios de início da aplicação dos subtestes tanto na versão original quanto na adaptada.

Nos subtestes Semelhanças, Compreensão, Dígitos e Armar O bjetos do WISC-III manteve-sea regra do manual original deiniciar a aplicação pelo item 1, independentemente da idade do sujeito. Considerando-se que nos subtestes Vocabulário e Arranjo de Figuras, exceto para 0 item 1, nenhum outro apresentou freqüência de $95 \%$ de acertos, também se estabeleceu esse item como o inicial para todas as idades. Pode-se concluir que, geralmente, o início da aplicação da versão adaptada ocorre em itens anteriores aos da versão original.

Enquanto no WISC-III observa-se diferentes critérios deinício da aplicação do subteste, segundo aidadeda criança, no WAIS-III há um critério único para todas as idades. Como pode ser observado na Tabela 3, no WAIS-III, dos oito subtestes que possuem critérios de início de aplicação apenas três deles sofreram alteração, a saber, Completar Figuras, Vocabulário e Semelhanças. Nesses dois últimos subtestes o início da aplicação passou a ser a partir do item 1, indicando que os itens na versão adaptada mostraram-se mais difíceis para a amostra brasileira investigada. Como conseqüência, o procedimento de aplicação dos itens

Tabela 3

Critérios deInício da Aplicação dos Subtestes no WISC-III eno WAIS-III nas Versões Original eAdaptada

\begin{tabular}{|c|c|c|c|}
\hline \multirow{13}{*}{$\begin{array}{l}\text { Teste } \\
\text { WISC-III }\end{array}$} & \multirow{2}{*}{ Subteste } & \multicolumn{2}{|c|}{ Critério deinício } \\
\hline & & Versão original* & Versão adaptada \\
\hline & \multirow{3}{*}{ Informação } & 8 a 10 anos: item 5 & 8 a 13 anos: item 85 \\
\hline & & 11 a 13 anos: item 8 & 14 a 16 anos: item 12 \\
\hline & & 14 a 16 anos: item 11 & \\
\hline & \multirow{4}{*}{ Aritmética } & 6 anos: item 1 & 6 e 7 anos: item 1 \\
\hline & & 7e 8 anos: item 6 & 8e 9 anos: item 8 \\
\hline & & 9 a 12 anos: item 12 & 10 a 13 anos: item 10 \\
\hline & & 13 a 16 anos: item 14 & 14 a 16 anos: item 13 \\
\hline & \multirow{4}{*}{ Vocabulário } & 6 a 8 anos: item 1 & \multirow{4}{*}{6 a 16 anos: item 1} \\
\hline & & 9e 10 anos: item 3 & \\
\hline & & 11 a 13 anos: item 5 & \\
\hline & & 14 a 16 anos: item 17 & \\
\hline \multirow[t]{10}{*}{ WAIS-III } & & & \multirow{7}{*}{$\begin{array}{l}6 \text { anos: item } 1 \\
7 \text { a } 9 \text { anos: item } 3 \\
10 \text { a } 16 \text { anos: item } 6 \\
6 \text { a } 16 \text { anos: item } 1\end{array}$} \\
\hline & \multirow{4}{*}{ Completar Figuras } & 6 e 7 anos: item 1 & \\
\hline & & 8a 9 anos: item 5 & \\
\hline & & 10 a 13 anos: item 7 & \\
\hline & & 14 a 16 anos: item 11 & \\
\hline & \multirow[t]{2}{*}{ Arranjo de Figuras } & 6 a 8 anos: item 1 & \\
\hline & & 9 a 16 anos: item 3 & \\
\hline & Completar Figuras & Item 6 & Item 3 \\
\hline & Vocabulário & Item 4 & Item 1 \\
\hline & Semelhanças & Item 6 & Item 1 \\
\hline
\end{tabular}

Nota. Fonte: $*$ WISC-III = Wechsler (1991); WAIS-III = Wechsler (1997). 
anteriores ao do início da aplicação não será utilizado em tais subtestes.

b) Critérios desuspensão

Na versão original do teste WISC-III, o critério utilizado para determinar o ponto de suspensão da aplicação dos itens em cada subteste foi a probabilidade de se obter acertos depois de vários pontos de interrupção examinados. Assim, em Vocabulário, por exemplo, foi concluído que depois de ocorrer quatro erros consecutivos, a probabilidade de acertar os demais itens seria muito pequena (Wechsler, 1991). Não tendo encontrado nenhuma outra referência mais detalhada sobre essa metodologia para estabelecer os critérios de suspensão, para a amostrabrasileira examinou-seo percentual de sujeitos que obtiveram acerto depois de cada um dos pontos de interrupções analisados nos subtestes Informação, Semelhanças, Aritmética, Vocabulário, Compreensão, Completar Figuras eArranjo de Figuras. Partindo-se do número de erros proposto no teste original para a suspensão de cada subteste, investigou-se o percentual de sujeitos queacertavam algum item após esseponto. Em Completar Figuras, por exemplo, após a seqüência de cinco erros consecutivos (critério do manual original), encontrou-se 186 participantes (23\% da amostra) com acerto(s) após este padrão de erro, cujos escores deveriam ser recodificados com zero no caso deste critério ser adotado. Considerando-se o número de casos elevados adotou-se como critério para suspensão da aplicação, aceitar um percentual de $5 \%$ deerros após o item de suspensão. Dessa forma, procurou-se, em cada subteste, o padrão de erros após o qual ocorria 5\% de possibilidade dealguém acertar algum item final.

No WAIS-III, os critérios de suspensão para os subtestes foram definidos após a análise da probabilidade de acerto em três possíveis critérios, sendo um anterior e outro posterior ao estabelecido para a versão original. Por exemplo, no subteste Completar Figuras o critério de suspensão original é de cinco erros consecutivos. Assim, testou-se tanto esse critério quanto o de quatro (anterior) e o de seis (posterior). Dada a impossibilidade de proceder algum tipo de comparação estatística entre as probabilidades obtidas por meio de uma função baseada na verossimilhança, optou-se por adotar os mesmos critérios da versão original americana em todos os subtestes (com exceção do Raciocínio Matricial), uma vez que as diferenças das probabilidades entre os critérios originais e os posteriores a eles foram muito pequenas. No subteste Raciocínio Matricial, o critério original de acerto após quatro erros consecutivos ou quatro erros em cinco itens consecutivos apresentou probabilidade de 0,23, enquanto no critério de apenas quatro erros consecutivos a probabilidade foi de 0,19. Desse modo, adotou-se esse último critério, por ser o que representou menor perda de informação.

A Tabela 4 apresenta os critérios de suspensão nasversões originais e adaptadas dos testes WISC-III e WAISIII.

Como pode ser observado na Tabela 4, com relação aos critérios originais de suspensão da aplicação dos subtestes, no WISC-III o ponto de corte utilizado na adaptação (5\%) tornou se mais leniente, uma vez que, exceto em Aritmética eCubos, para todos os demais subtestes o número de erros consecutivos estabelecidos parainterromper a aplicação ficou superior ao original. Como conseqüência, o tempo despendido para a administração do teste pode tornar-se maior, mas por outro lado minimiza-se a possibilidade da ocorrência de acertos após o ponto de interrupção da aplicação do subteste, possibilitando maior expressão do domínio de conhecimento da criança.

Tabela 4

Critérios de Suspensão da A plicação dos Subtestesno WISC-III eno WAIS-III nas Versões Original eA daptada

\begin{tabular}{llcc}
\hline \multirow{2}{*}{ Teste } & Subteste & \multicolumn{2}{c}{ Critério de suspensão } \\
\hline \multirow{2}{*}{ WISC-III } & Informação & 5 & Versão original* \\
& Semelhanças & 4 & 8 \\
& Vocabulário & 4 & 8 \\
& Compreensão & 3 & 6 \\
& Completar Figuras & 5 & 6 \\
& Arranjo de Figuras & 3 & 6 \\
\hline \multirow{2}{*}{ WAIS-III } & Raciocínio Matricial & 4 erros consecutivos ou 4 & \multirow{2}{*}{4 erros consecutivos } \\
& & erros em 5 itens consecutivos & \\
\hline
\end{tabular}

Nota. Fonte: $*$ WISC-III = Wechsler (1991); WAIS-III = Wechsler (1997). 
Tabela 5

Grupos Etários Considerados para o Estabelecimento das N ormas por Idadenas Versões Americana eAdaptada do WISC-III edo WAIS-III

\begin{tabular}{|c|c|c|}
\hline Teste & Versão & Grupos etários \\
\hline \multirow[t]{2}{*}{ W ISC-III } & Original $^{1}$ & $\begin{array}{l}33 \text { grupos etários: } 6 \text { anos e } 0 \text { meses a } 16 \text { anos e } 11 \text { meses, com faixa de } 4 \text { meses } \\
\text { em cada grupo }\end{array}$ \\
\hline & Adaptada & 06 grupos etários: 6 anos; 7 anos; 8-9; 10-11; 12-13 e 14-16 anos \\
\hline \multirow[t]{2}{*}{ WAIS-III } & Original $^{2}$ & $\begin{array}{l}13 \text { grupos etários: 16-17; 18-19; 20-24; 25-29; 30-34; 35-44; 45-54; 55-64; 65-69; } \\
\text { 70-74; 75-79; 80-84 e 85-89 anos }\end{array}$ \\
\hline & Adaptada & 08 grupos etários: 16-17; 18-19; 20-29; 30-39; 40-49; 50-59; 60-64 e 65-89 anos \\
\hline
\end{tabular}

Nota. Fonte: 1 = Wechsler (1991); 2 = Wechsler (1997).

\section{Grupos etários para o estabelecimento de normas}

Além das alterações supracitadas nos procedimentos de aplicação e correção dos testes WISC-III e WAIS-III, cabe destacar também que em relação às normas por idade houve uma redução no número de grupos etários para os quais foram estabelecidas. Essa simplificação foi decorrente dos limites das amostras delineadas para as pesquisas de adaptação dos respectivos testes.

A Tabela 5 evidencia os grupos etários considerados para a elaboração das normas americanas e brasileiras.

Como pode ser observado na Tabela 5, as faixas etárias nas versões adaptadas foram condensadas. Dada a importância que representa a existência de normas por idade contemplando faixas etárias menores para fins de avaliação do funcionamento intelectual, a expansão das amostras para normatização será objeto de estudos futuras das pesquisadoras.

\section{Discussão}

A tradução de um teste psicológico e a elaboração das normas para um novo contexto não efetivam o processo de adaptação evalidação do instrumento. Além dos estudos dascaracterísticas psicométricas, são procedimentos essenciais a atualização dos itens, bem como a revisão dos processos de aplicação e de correção. Na adaptação dos testes WISCIII eWAIS-III não houvenenhuma alteração substancial no formato do instrumento. As modificações mais relevantes referiram-se à ordenação e ao conteúdo de alguns itens. Tanto no WISC-III quanto no WAIS-III foi necessária a reordenação na seqüência dositens na maioria dos subtestes e algumas adaptações para eliminar 0 viés cultural, principalmente, no subteste Informação. No WISC-III, os critérios de início e suspensão dos subtestes alteraram-se mais do que no WAIS-III, da mesma forma que os bônus pela execução correta e rápida dos itens.
Considerando-seas evidências das alterações no processo de adaptação do WISC-III e do WAIS-III, pode-se concluir que a utilização de um instrumento estrangeiro sem a sua devida adaptação pode colocar em risco a validade e precisão de avaliações efetuadas quando não se leva em conta as características socioeconômicas e culturais das amostras em que os testes serão utilizados.

Embora as diretrizes preconizadas pela Comissão Internacional de Testes relacionadasao processo deadaptação constituam em fontes imprescindíveis para 0 desenvolvimento de pesquisas voltadas para tal finalidade, ao desenvolver as pesquisas de adaptação do WISC-III e do WAIS-III, as autoras depararam com a escassez de referências práticas sobre os procedimentos e análises necessárias para 0 estabelecimento de muitos dos aspectos envolvidos na construção e adaptação de instrumentos psicológicos. Deste modo, o presente artigo teve como objetivo relatar também os procedimentos adotados para a adaptação dos referidos testes, o que poderá servir como referência para pesquisas futuras.

\section{Referências}

Figueiredo, V. L. M. (2001). Uma adaptação brasileira do teste de inteligência WISC- III. Tese de Doutorado não-publicada, Curso de Pós-graduação em Psicologia, Universidade de Brasília. Brasília, DF.

Flynn, J. R. (1987). Massive IQ gains in 14 nations: What IQ tests really measure. Psychological Bulletin, 101(2), 171-191.

Hambleton, R. K. (1993). Translating achievement tests for use in crossnational studies. Europeen Journal of Psychological Assessment, 9(1), 57-68.

Hambleton, R. K. (1994). Guidelines for adapting educational and psychological tests: A progress report. European Journal of Psychological Assessment, 10(3), 229-244.

Hambleton, R. K. \& Bollwark, J. (1991). Adapting tests for use in different cultures: Technical issues and methods. ITC Bulletin/ Bulletin CIT: International Tet Bulletin Issues, 32/33, 3-32.

Muñiz, J. \& Hambleton, R. K. (1996). Diretrizes para a tradução e adaptação de testes. Papeles del psiólogo: Revista del olegio oficial de psiólogos. Época III, $66,63-70$. 
Nascimento, E. (2000). Adaptação e validação do teste WAIS-III para um ontexto brasileiro. Tese de Doutorado não-publicada, Curso de Pós-graduação em Psicologia, Universidade de Brasília.Brasília, DF.

The Psychological Corporation (1997). WAIS-III and WMS-III: Technical manual. San Antonio, DC: Autor.

Van de Vijver, F. \& Hambleton, R. K. (1996). Translating tests: Some practical guidelines. European Psychologist, 1(2), 89-99.

Wechsler, D. (1944). The measurement of adult intelligenœ ( $3 \stackrel{a}{a}$ ed.). Baltimore: The Williams \& Wilkins.

Wechsler, D. (1991). Wechsler Intelligence Scale for Children (3a ed.) (WISC-III): Manual. San Antonio: Psychological Corporation.
Wechsler, D. (1994). Test de inteligencia para niños WISC III. Buenos Aires: Paidós.

Wechsler, D. (1997). WAIS-III: Administration and sooring manual. San Antonio: The Psychological Corporation.

Sobre os autores

Elizabeth do Nascimento éProfessora do Departamento dePsicologia da UniversidadeFederal de Minas Gerais, Mestre pela Universidade Federal de Minas Gerais (UFMG) eD outora em Psicologia pela Universidade de Brasília (UnB), Coordenadora do Laboratório de Avaliação das Diferenças Individuais (LADI) do Departamento de Psicologia/UFMG.

Vera Lúcia Marques de Figueiredo é Professora do curso de Graduação em Psicologia e do Mestrado em SaúdeeComportamento da UniversidadeCatólica dePelotas-RS, Mestrepela Pontifícia Universidade Católica do Rio Grande do Sul (PUCRS) e Doutora pela Universidade de Brasília (UnB). 